\title{
Maternal and Fetal Outcome in Intrahepatic Cholestasis of Pregnancy in a Multicultural Society Conducted at a Tertiary Care Hospital in Dubai
}

\author{
Nighat Aftaba,b Saima Faraz ${ }^{a, b} \quad$ Komal Hazaric Faiza Badawi Mahgouba, b \\ aObstetrics and Gynecology Department, Latifa Women and Children Hospital, Dubai, UAE; bepartment of \\ Obstetrics and Gynecology, Dubai Medical College, Dubai, UAE; 'Internal Medicine Department, Latifa Women and \\ Children Hospital, Dubai, UAE
}

\section{Keywords}

Maternal-fetal outcomes · Intrahepatic cholestasis of pregnancy $\cdot$ Bile acids

\begin{abstract}
Introduction: Intrahepatic cholestasis of pregnancy (ICP) has been sparsely studied especially in the Middle East. The incidence and outcome of ICP varies worldwide. Its incidence in the Middle East and primary maternal and fetal outcome must be evaluated to ascertain the burden so that appropriate preventive and intervention measures can be formulated and implemented. Objective: To assess the incidence, associations, and maternal-fetal outcomes in ICP. Design: Case-control study. Settings: tertiary care hospital settings affiliated with the academic center in the UAE. Patients and methods: a total of 150 patients were included from October 2016 to September 2018 in the study with 75 cases of ICP and 75 controls matched according to age and date of delivery. The maternal risk factors attributable to ICP were recorded. Biochemical profile of mothers was monitored. Maternal and fetal outcomes were compared in 2 groups. Main outcomes measured: incidence and associations of ICP were evaluated. Maternal and fetal outcomes were compared in cases and controls. Sample size: 150. $\boldsymbol{R e}$ sult: The incidence of ICP in our study in the UAE was $1.0 \%$.
\end{abstract}

ICP has significant association with past obstetric cholestasis history ( $p$ value $<0.01$, odds ratio [OR] 9.3, 95\% Cl: 2.1-41.8), gestational diabetes ( $p$ value $<0.05$, OR 2.0, 95\% Cl: 1.0-3.8), pre-eclampsia ( $p$ value $<0.05$, OR $7.2,95 \% \mathrm{Cl}: 1.6-33.1$ ), and undergoing induction of labor ( $p$ value $<0.01$, OR $8.1,95 \% \mathrm{Cl}$ : 3.7-17.8). The maternal bile acid level above $40 \mu \mathrm{mol} / \mathrm{L}$ is associated with higher chances of preterm delivery ( $p$ value $<0.01$, OR $8.2,95 \% \mathrm{Cl}$ : 3.0-22.5), intrauterine fetal demise ( $p$ value $<0.01$ ), low birth weight ( $p$ value $<0.01$, OR 13.6, 95\% $\mathrm{Cl}: 4.2-43.5$ ), respiratory distress ( $p$ value $<0.05$, OR $15.5,95 \%$ Cl: 1.8-132.7), poor Apgar score ( $p$ value $<0.05$, OR 12.720, $95 \%$ Cl: 1.5-111.4), and NICU admissions ( $p$ value $<0.01$, OR 9.0, 95\% Cl: 1.8-45.9). Conclusion: ICP mothers have low incidence in the UAE and significant association with gestational diabetes and pre-eclampsia. High maternal bile acids above $40 \mu \mathrm{mol} / \mathrm{L}$ have poor fetal outcomes.

(c) 2021 The Author(s).
Published by S. Karger AG, Basel

\section{Introduction}

Pregnancy is a condition of metabolic and anatomic stress which alters mother physiology considerably. There are physiological and biochemical changes in liver functions which are needed to support the growing fetus. Any abnormal change needs to be differentiated from physi- karger@karger.com www.karger.com/dmj

Karger $\frac{1}{\%}$

BOPEN ACCESS
(C) 2021 The Author(s)

Published by S. Karger AG, Basel

This article is licensed under the Creative Commons AttributionNonCommercial-NoDerivatives 4.0 International License (CC BYNC-ND) (http://www.karger.com/Services/OpenAccessLicense). Usage and distribution for commercial purposes as well as any distribution of modified material requires written permission.
Saima Faraz

Specialist, Obstetrics and Gynecology Department

Latifa Women and Children Hospital, PO Box 9115

Oud Metha Road, Al Jadaf, Dubai 4545 (United Arab Emirates)

saimafaraz42@gmail.com 
ological change as liver dysfunction has adverse implications on the mother and fetus [1]. Jaundice and pruritus are few of the manifestations of liver disease and occur in 1 in 2000 pregnancies [2]. There are many causes of liver diseases, and some disorders are specific to pregnancies as intrahepatic cholestasis while others may coincidently occur with pregnancy as infective hepatitis [3].

Intrahepatic cholestasis of pregnancy (ICP) is among the common diseases of the liver during pregnancy with an incidence of $0.4-15 \%$ all over the world; however, there are some regional discrepancies. It is more common in South America (10\%) and less common in Europe $(0.4 \%)$ [4]. This large difference is attributed largely to environmental factors. It generally follows a benign course, and the patient has unremarkable recovery with no permanent liver damage [3]. The symptoms primarily start by the end of second or early third trimester with pruritus as a common complaint followed by elevated bile acids (BA) and liver enzymes. The liver functions and symptoms return to normal within 6 weeks after delivery [5]. The pathogenesis of ICP is believed to be multifactorial, and the exact mechanism is not known. Hormonal changes during pregnancy with elevated estrogen and altered cell membrane configuration in bile ducts and liver cells along with defected transport mechanisms in biliary canaliculi indicate a strong genetic cause [6]. However, geographic distribution and change in incidence with weather also point out environmental influencers [7].

The maternal and fetal consequences in ICP have been studied in various populations. A study done in Sweden showed that women with ICP have almost 3 times more chances of developing gestational diabetes, pre-eclampsia, and preterm labor [8]. However, other studies predict a benign maternal outcome with increased postpartum hemorrhage and vitamin $\mathrm{K}$ deficiency only in severe persistent ICP with high levels ( $>40$ $\mu \mathrm{mol} / \mathrm{L}$ ) of circulating BA [4]. There are various studies which predict fetal complications as well. A study done in Nepal showed that there were high chances of meconium aspiration (32.5\%), fetal distress $(26.5 \%)$, admission in neonatal intensive care unit (NICU) $(48.8 \%)$, and high mortality (12.5\%) in cases of ICP [9]. Others have correlated maternal serum BA levels with fetal outcomes. There were progressive higher odds of still birth as level of bile salts increases beyond $40 \mu \mathrm{mol} / \mathrm{L}$ [10]. On the contrary, a study done in USA showed no association of mother's biochemical markers with fetal complications till the BA concentration is above $100 \mu \mathrm{mol} / \mathrm{L}$. In fact, ICP in previous pregnancies was associated with less fetal problems [11].
The rationale of our study is to describe the associations and maternal-fetal outcomes associated with ICP in a multicultural society in the United Arab Emirates in a particular setting. Our second aim was to determine if significant clinical and biochemical predictors of morbid fetal outcome correlate well in mothers diagnosed with ICP. To our knowledge, this is the first study being conducted in the Middle East and would help us understand the impact of ICP in this region.

\section{Patients and Methods}

This was a case-control study conducted at a tertiary health facility affiliated with the academic center (Dubai, United Arab Emirates). All procedures were in accordance with the ethical standards of the Institutional Research Committee, the National Research Committee, and the 1964 Helsinki Declaration and its later amendments. Written informed consent was taken from all subjects.

The sample size was calculated by using the World Health Organization calculator keeping a confidence interval of $95 \%$, and proportion of exposed cases for respiratory distress was $17.1 \%$, whereas the percent of exposed controls was 4.6\% [12]. A total of 150 patients were inducted in the study, and the ratio of cases and controls was 1:1. The sampling was done by the nonrandom consecutive method by retrieving records from the central database. All the data were extra plotted in a predesignated proforma. All the booked pregnant patients diagnosed with obstetric cholestasis and delivered from October 1, 2016, to September 30, 2018, were recruited. ICP was defined as pregnancies in the late 2 nd or 3 rd trimester presenting with symptoms of itching and raised BA $(>10$ $\mu \mathrm{mol} / \mathrm{L}$ ) and deranged liver function tests. The controls were taken randomly as cases of pregnancy in the late 2 nd or 3 rd trimester with no known cholestasis or liver disease. The controls were matched for age, date of delivery, and parity with cases. The pregnant females who were diagnosed with chronic liver disease owing to infective hepatitis (B and C), congenital liver diseases as Wilsons disease and primary biliary cirrhosis, cytomegalovirus, Epstein-Barr virus, symptomatic gall stones and cholecystitis, acute fatty liver of pregnancy, and HELLP syndrome were excluded from the study.

All the patients were booked, diagnosed, and followed up throughout pregnancy, at delivery, and up to 6 weeks postnatal. Laboratory tests were repeated at day 10 and 6 weeks after delivery. The data which were collected for cases and controls included demographic details, ethnic background, gravidity, parity, BMI, history of ICP in previous pregnancy, history of biliary and liver disease, spontaneous or induced labor, mode of delivery, and any complications like gestational diabetes, pre-eclampsia, and postpartum hemorrhage. The biochemical workup of patients included a maximum value of serum aspartate transaminase (AST), alanine transaminase (ALT), total bilirubin, and BA. Serum liver enzymes and BA were determined using routine laboratory analyzers. The fetal outcomes studied were still births, birth weight, Apgar score at time of delivery and after $5 \mathrm{~min}$, fetal distress, major congenital anomalies, hyperbilirubinemia, meconium staining of amniotic fluid at delivery, meconium aspiration, pneumonia, respiratory distress, and sepsis. Fetal distress was continuous deterioration of the fetus or a fetal heart rate of $<110$ beats per minute lasting 3 min 
Table 1. Sociodemographic characteristics of the mother in cases and controls $(n=150)$

\begin{tabular}{|c|c|c|c|c|c|}
\hline S. No. & Demographic characteristics & ICP cases & Controls & $p$ value & Odds ratio and $95 \% \mathrm{CI}$ \\
\hline 1 & Age, years & $31.73 \pm 5.92$ & $32.26 \pm 5.08$ & 0.538 & - \\
\hline 3 & Gestational age at delivery (<37 wks) & $31(41.1 \%)$ & $24(32 \%)$ & 0.204 & $1.508(0.799-2.844)$ \\
\hline 4 & $\begin{array}{l}\text { Current pregnancy complications } \\
\text { Gestational diabetes } \\
\text { Pre-eclampsia }\end{array}$ & $\begin{array}{l}27(36 \%) \\
8(10.7 \%)\end{array}$ & $\begin{array}{r}18(24 \%) \\
2(2.7 \%)\end{array}$ & $\begin{array}{l}0.047 \\
0.01\end{array}$ & $\begin{array}{l}1.967(1.010-3.831) \\
7.222(1.576-33.100)\end{array}$ \\
\hline 5 & Positive past obstetric cholestasis history & $16(21.3 \%)$ & $2(2.7 \%)$ & 0.004 & $9.275(2.06-41.771)$ \\
\hline 6 & Past IUFD history & $2(2.7 \%)$ & $2(2.7 \%)$ & 0.971 & $0.964(0.133-7.008)$ \\
\hline 7 & IVF pregnancy & $7(9.3 \%)$ & $5(6.7 \%)$ & 0.200 & $0.548(0.217-1.386)$ \\
\hline 9 & $\begin{array}{l}\text { Parity } \\
\quad \text { Primigravida } \\
\quad 2-4 \\
>4\end{array}$ & $\begin{array}{l}24(32 \%) \\
46(61.3 \%) \\
5(6.6 \%)\end{array}$ & $\begin{array}{r}23(30.7 \%) \\
44(58.7 \%) \\
8(10.7 \%)\end{array}$ & $\begin{array}{l}0.469 \\
0.597 \\
-\end{array}$ & $\begin{array}{l}2.480(0.212-28.958) \\
1.927(0.170-21.890) \\
-\end{array}$ \\
\hline 10 & $\begin{array}{l}\text { Ethnic background } \\
\text { Arabs-Middle East } \\
\text { Asia } \\
\text { Africa } \\
\text { UK/Europe }\end{array}$ & $\begin{array}{c}53(70.7 \%) \\
16(21.3 \%) \\
4(5.3 \%) \\
2(2.7 \%)\end{array}$ & $\begin{aligned} & 49(65.3 \%) \\
& 17(22.7 \%) \\
& 8(10.7 \%) \\
& 1(1.3 \%)\end{aligned}$ & $\begin{array}{l}0.412 \\
0.296 \\
0.142 \\
-\end{array}$ & $\begin{array}{l}0.383(0.039-3.788) \\
0.286(0.028-3.142) \\
0.148(0.012-1.900) \\
-\end{array}$ \\
\hline
\end{tabular}

ICP, intrahepatic cholestasis of pregnancy; IUFD, intrauterine fetal demise.

or longer, requiring emergent delivery. Respiratory distress was defined as any neonate that required invasive or noninvasive ventilation after delivery, diagnosis of pneumonia, documentation of respiratory distress secondary to meconium aspiration, or documentation by a health care provider based on physical examination.

Data were entered and analyzed using IBM Statistical Package of Social Sciences 21.0. Quantitative variables like age, serum ALT, AST, and BA, and fetal weight were represented in mean and standard deviation. Qualitative variables such as ethnic background, parity, and fetal complications were shown as frequency and percentages. The data were represented in graphs. Student's $t$ test was used to assess the $p$ value for quantitative variables in both cases and controls. The $\chi^{2}$ test was used for determining significance of qualitative variables in both groups. Logistic and multinomial regression was used to determine odds ratio and 95\% confidence interval. $p$ value $<0.05$ was taken as significant.

\section{Results}

A total of 150 patients were included in the study, 75 cases were diagnosed with ICP and 75 were controls among 7,113 pregnant cases that reported in Latifa Hos- pital (Dubai, United Arab Emirates) making 1.0\% incidence $(75 / 7,113)$. The mean maternal age among cases was $31.7 \pm 5.9$ years, whereas it was $32.3 \pm 5.1$ years in the control group ( $p$ value $=0.5$ ). The sociodemographic characteristics of mothers in cases and controls are given in Table 1. The mean gestational age at time of delivery in cases was $36 \pm 4.1$ weeks, whereas in controls, it was $37 \pm$ 2.9 weeks ( $p$ value $=0.2$ ). The mean gestational age at time of diagnosis of ICP was $225 \pm 37$ days. There was no statistical significance in terms of age and BMI in both groups.

The maternal and fetal outcomes for cases and controls are depicted in Tables 2 and 3, respectively. There is no statistically significant difference in fetal outcome when cases and controls were compared except in terms of NICU admission. However, Table 3 shows that when BA levels exceed $40 \mu \mathrm{mol} / \mathrm{L}$, there is statistically significant risk to the fetus in terms of intrauterine fetal demise (IUFD), low birth weight, respiratory distress, and preterm delivery. 
Table 2. Peripartum outcomes in both cases and controls $(n=150)$

\begin{tabular}{llllll}
\hline S. No. & Variable & ICP cases & Controls & $p$ value & Odds ratio and 95\% CI \\
\hline 1 & Induction of labor & $34(45.3 \%)$ & $10(13.3 \%)$ & $<0.001$ & $8.100(3.688-17.789)$ \\
\hline 2 & Mode of delivery & Spontaneous & & & \\
& Elective C-section & $32(42.6 \%)$ & $30(40 \%)$ & 0.980 & $1.010(0.465-2.193)$ \\
& Emergency C-section & $25(33.3 \%)$ & $27(36 \%)$ & 0.905 & $0.952(0.428-2.119)$ \\
\hline 3 & $18(24 \%)$ & $18(24 \%)$ & - & - \\
\hline 4 & Preterm delivery & $27(36 \%)$ & $20(26.7 \%)$ & 0.116 & $1.660(0.882-3.125)$ \\
\hline 5 & PPH & $11(14.7 \%)$ & $7(9.3 \%)$ & 0.696 & $1.206(0.472-3.082)$ \\
\hline
\end{tabular}

ICP, intrahepatic cholestasis of pregnancy.

Table 3. Fetal outcomes in both cases and controls $(n=167)$

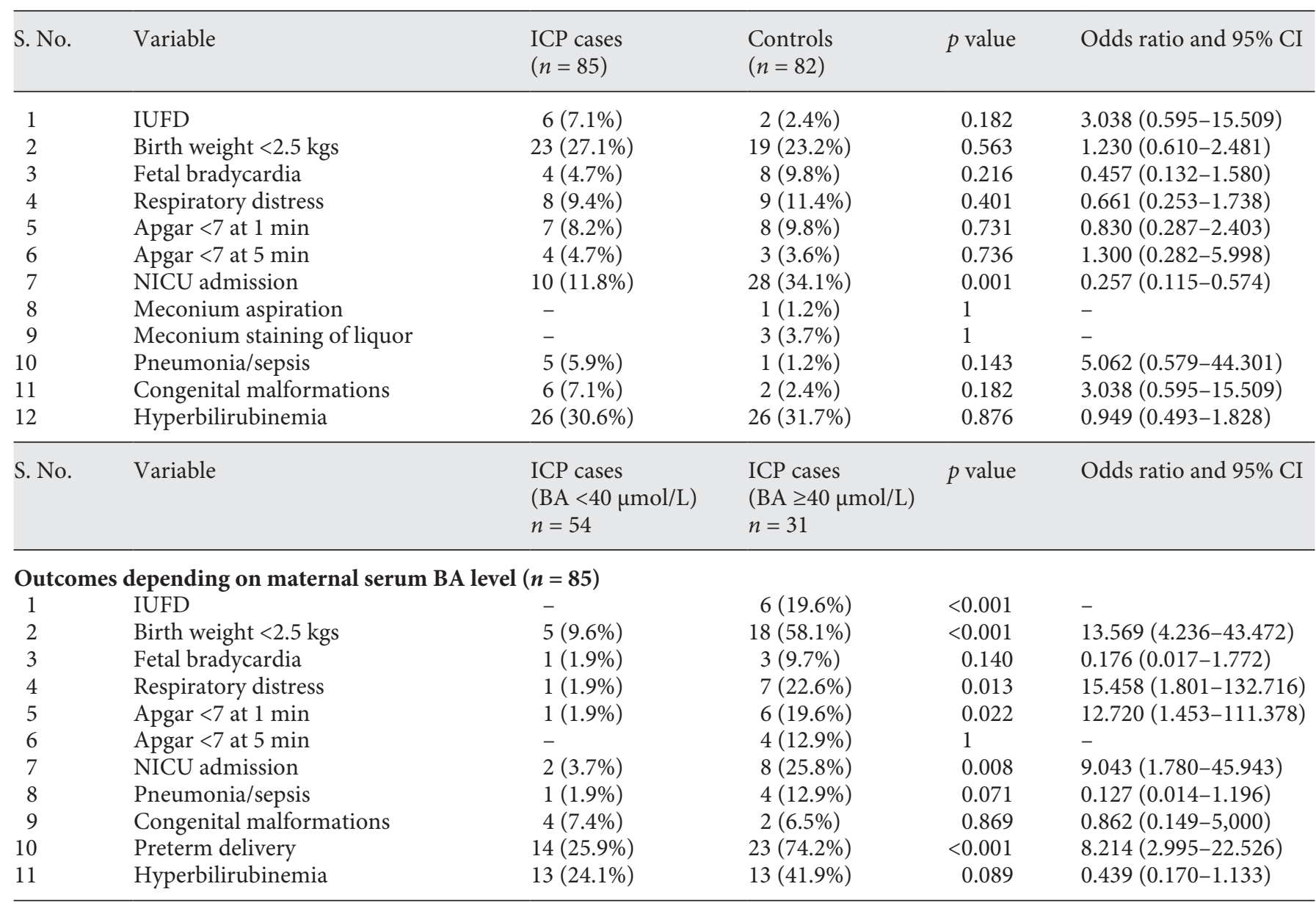

ICP, intrahepatic cholestasis of pregnancy; BA, bile acids; IUFD, intrauterine fetal demise; NICU, neonatal intensive care unit. 
Table 4. Biochemical profile of cases and controls $(n=150)$

\begin{tabular}{|c|c|c|c|c|}
\hline \multirow[t]{2}{*}{ S. No. } & \multirow[t]{2}{*}{ Biochemical profile } & \multicolumn{2}{|c|}{ Median \pm interquartile range } & \multirow[t]{2}{*}{$p$ value } \\
\hline & & ICP cases & Controls & \\
\hline 1 & ALT, IU/L & $85 \pm 104$ & $12 \pm 7$ & $<0.001$ \\
\hline 2 & AST, IU/L & $44 \pm 58$ & $13 \pm 7$ & $<0.001$ \\
\hline 3 & $\mathrm{BA}, \mu \mathrm{mol} / \mathrm{L}$ & $24 \pm 41.3$ & - & - \\
\hline
\end{tabular}

ICP, intrahepatic cholestasis of pregnancy; AST, aspartate transaminase; ALT, alanine transaminase; BA, bile acids.

The biochemical profile of cases and controls showed significant difference when values of ALT and AST were compared by $p$ value $<0.001$ in both cases. This is shown in Table 4 along with Figure 1 showing distribution of values. The main symptom was itching in palms and soles occurring in $72(96 \%)$ cases followed by pruritic rash in $45(60 \%)$ patients and scratch marks in $30(40 \%)$, whereas jaundice was not present in any case. Only 22 (29.3\%) had symptoms severe enough to be treated with ursodeoxycholic acid. Rest of the patients received only symptomatic therapy where indicated.

\section{Discussion}

There is paucity of data about ICP and its fetal and maternal outcomes in the Middle East. The incidence of ICP calculated in our study was $1.0 \%$. This is comparable to another regional study conducted in Nepal (1.1\%) [9]. However, the incidence in a Swedish study was $0.5 \%$ [2, 8]. The sample population was from multiple ethnic backgrounds and did not show any statistical significance or increased incidence among a particular race when compared with controls ( $p$ value $=0.2)$. Rook et al. [11] in their study showed that Latino women have higher odds of getting ICP than normal American population (OR 4.9, 95\% CI: 2.5-9.51, $p<0.01$ ). However, the study by Geenes and colleagues [13] did not show any racial preference in terms of ICP. The mean age at time of diagnosis in our study was $31.7 \pm 5.9$. The mean age was 29.6 \pm 6.3 in a study done in UK [13]. Similarly, a study in Northern India had a mean age of 29.88 [14]. The mean BMI in our study was also much higher when compared with similar ICP studies [11-13]. However, a meta-analysis of ICP patients showed that they had mean $1.6 \mathrm{~kg} / \mathrm{m}^{2}$ more BMI than controls [10]. The difference in BMI and age can be related to transition of developed countries towards obesity especially in the Middle East [15]. While assessing sociodemographic factors of the mother, the only statistically significant point attributed to ICP was past obstetric cholestasis history ( $p$ value $<0.01$, OR 9.3, 95\% CI: 2.1-41.8). Past history of IUFD, mode of conception, parity, and previous miscarriages were not significantly associated with ICP patients in our observation. Arthuis et al. [12] found out that family history and history of ICP in previous pregnancy were significantly associated with occurrence of ICP ( $p$ value $<0.01$ ). A study by Ovadia et al. [10] showed that singleton ICP pregnancies are more prone to develop complications. Similarly, various studies showed that IVF-induced pregnancies have higher tendencies to develop ICP with significant complications $[7,16]$.

One significant finding in our study was that the patients of ICP had 2 times more chances of acquiring gestational diabetes ( $p$ value $<0.05$ ) and 7 times more chances of having pre-eclampsia ( $p$ value $<0.01$ ) during their pregnancies. This is coherent with data published in the international literature $[10,17,18]$. The mean onset of symptoms in our cases was $32.1 \pm 5.3$. Brouwers and his colleagues [19] found out that diagnostic symptoms appeared between 33rd and 36th. Pruritus in palms and soles was the most dominant symptom, and there was no clinically significant jaundice noted in our study. Similar findings were noted by Ghimire SP et al. [9] and Kant et al. [14] in their studies. The women with ICP had elevated ALT and AST when compared with controls ( $p$ value $<0.01)$. In addition to liver enzymes, BA were significantly raised in cases. The rise in liver enzymes along with BA is reported in various studies and constitutes a diagnostic criterion for ICP [20].

The mothers with ICP had a significantly higher odds of undergoing induction of labor ( $p$ value $<0.01$, OR 8.1, 95\% CI: 3.7-17.8). Such high association can be attributed to our hospital's policy of close observation and proactive approach towards management of ICP patients. This active induction method has shown favorable out- 

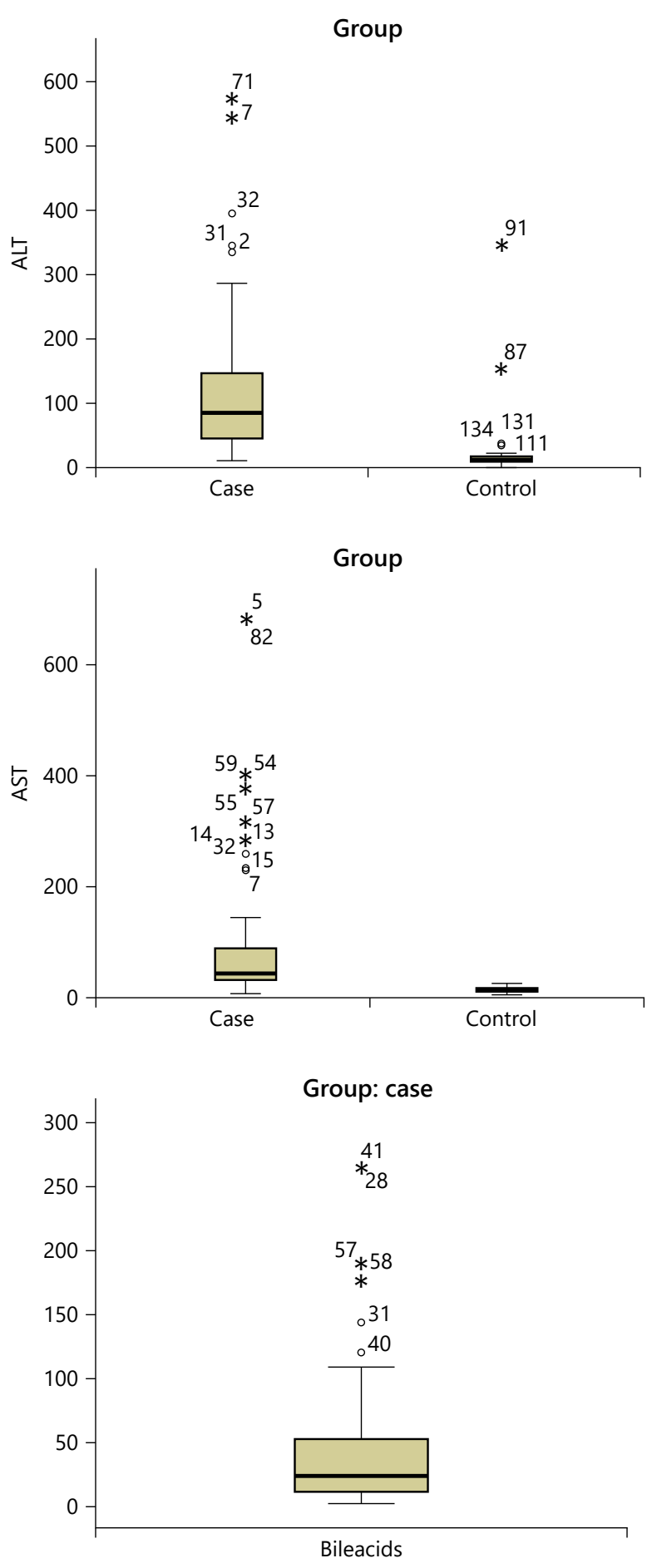

Fig. 1. Range of ALT, AST, and BA in cases and controls. AST, aspartate transaminase; ALT, alanine transaminase; BA, bile acids. comes for the mother and child. No significant relationship was established in terms of still birth, mode of delivery, $\mathrm{PPH}$, and preterm births between the cases and controls. Two large studies by Shemer et al. [8] and Marathe et al. [18] also reported similar results with no still births and higher rates of induction in ICP cases $[8,18]$. The American College of Obstetrician and Gynecologists also recommends active labor management of ICP [21]. It has also been shown that early induction did not lead to any still birth in these cases. On the contrary, a large retrospective UK-based study pointed out a significant association of still birth and preterm delivery in cholestatic pregnancies [13]. Ghimire SP and companions [9] also noted $48 \%$ of their ICP pregnancies underwent caesarean sections, whereas the surgical intervention was comparable between the 2 groups in our study.

All the poor fetal outcomes compared in our study were not significantly related to ICP (Table 3 ). In fact, babies born to ICP mothers in our hospital had a lesser chance of NICU admission as compared to controls ( $p$ value $<0.01$, OR $0.3,95 \%$ CI: 0.1-0.6). A study done by Mullally and Hansen [22] showed that congenital malformations and abortions had no association with ICP, and birth weight of babies born to such mothers was also sufficient. However, different studies reported an increased incidence of meconium staining of amniotic fluid (45\%), fetal distress (22\%), and preterm delivery $(44 \%)[23,24]$. However, it was deduced that as the maternal BA increase especially above 40 $\mu \mathrm{mol} / \mathrm{L}$, the chances of preterm delivery, IUFD, poor Apgar score, and NICU admissions increase significantly. A large case-control study in the UK showed that women with severe ICP (based on BA levels) had significantly high chances of preterm delivery (OR 5.3, 95\% CI: 4.1-6.9), still birth (OR 2.5, 95\% CI: 1.0-6.4), and NICU admissions (OR 2.6, 95\% CI: 1.9-3.6) [13]. There were a few limitations in our study. It was done in a single center, so its findings cannot be generalized to the whole population. The number of controls was also small. We recommend a multicentric large randomized trial to ascertain the true causal relationship between ICP and feto-maternal outcomes.

\section{Conclusion}

ICP has low incidence rate in the UAE and is significantly associated with previous history of obstetric cholestasis, gestational diabetes, and pre-eclampsia. There are more chances of mothers with ICP to undergo induction of labor. Fetal outcome is dependent on levels of maternal BA and associated with higher chances of preterm 
delivery, IUFD, low birth weight, respiratory distress, poor Apgar score, and NICU admissions. However, with close monitoring, the chances of still birth can be averted.

\section{Acknowledgements}

The authors are extremely indebted to the Department of Pathology for timely provision of all laboratory investigations.

\section{Statement of Ethics}

Subjects (or their parents or guardians) have given their written informed consent, and the study protocol was approved by the Dubai Scientific Research Ethics Committee (DSREC), Dubai Health Authority, DSREC-03/2019_09 dated July 09, 2019.

\section{Conflict of Interest Statement}

The authors have no conflicts of interest to disclose.

\section{Funding Sources}

The authors did not receive any funding.

\section{Author Contributions}

N.A. conceived, designed, collected data, and did statistical analysis. S.F. and K.H. did data collection and manuscript writing. F.B.M. supervised the whole study and edited the manuscript.

\section{References}

1 Suresha I, Vijaykumar TR, Nandeesh HP. Predictors of fetal and maternal outcome in the crucible of hepatic dysfunction during pregnancy. Gastroenterol Res. 2017;10(1): 21-7.

2 Oladokun A, Otegbayo JA, Adeniyi AA. Maternal and fetal outcomes of jaundice in pregnancy at the University College Hospital, Ibadan. Niger J Clin Pract. 2009;12(3):277.

3 Mitta P, Rao SV. Fetomaternal outcome in jaundice complicating pregnancy. IOSR J Dent Med Sci. 2016;15(10):72-6.

4 Ozkan S, Ceylan Y, Ozkan OV, Yildirim S. Review of a challenging clinical issue: intrahepatic cholestasis of pregnancy. World J Gastroenterol. 2015 Jun 21;21(23):7134.

5 Estiú MC, Frailuna MA, Otero C, Dericco M, Williamson C, Marin JJG, et al. Relationship between early onset severe intrahepatic cholestasis of pregnancy and higher risk of meconium-stained fluid. PloS One. 2017;12(4): e0176504.

6 Williamson C, Geenes V. Intrahepatic cholestasis of pregnancy. Obstet Gynecol. 2014; 124(1):120-33.

7 Feng C, Li W-J, He R-H, Sun X-W, Wang G, Wang L-Q. Impacts of different methods of conception on the perinatal outcome of intrahepatic cholestasis of pregnancy in twin pregnancies. Sci Rep. 2018 Mar 5;8(1):1-8.

8 Wikström Shemer E, Marschall HU, Ludvigsson JF, Stephansson O. Intrahepatic cholestasis of pregnancy and associated adverse pregnancy and fetal outcomes: a 12-year population-based cohort study. BJOG. 2013 May; 120(6):717-23.

9 Ghimire SP, Ghimire A, Jha GS, Chhetry M, Kumar M. Feto-maternal outcomes in intra- hepatic cholestasis in pregnancy in a tertiary care centre in Eastern Nepal. J Nobel Med Coll. 2016 Sep 23;5(1):20-5.

10 Ovadia C, Seed PT, Sklavounos A, Geenes V, Di Ilio C, Chambers J, et al. Association of adverse perinatal outcomes of intrahepatic cholestasis of pregnancy with biochemical markers: results of aggregate and individual patient data meta-analyses. The Lancet. 2019 Mar 2; 393(10174):899-909.

11 Rook M, Vargas J, Caughey A, Bacchetti P, Rosenthal P, Bull L. Fetal outcomes in pregnancies complicated by intrahepatic cholestasis of pregnancy in a Northern California cohort. PLoS One. 2012;7(3):e28343.

12 Arthuis C, Diguisto C, Lorphelin H, Dochez V, Simon E, Perrotin F, et al. Perinatal outcomes of intrahepatic cholestasis during pregnancy: an 8-year case-control study. PLoS One. 2020 Feb 19;15(2):e0228213.

13 Geenes V, Chappell LC, Seed PT, Steer PJ, Knight M, Williamson C. Association of severe intrahepatic cholestasis of pregnancy with adverse pregnancy outcomes: a prospective population-based case-control study. Hepatology. 2014;59(4):1482-91.

14 Kant A, Goswami S, Gupta U, Razdan A, Amle D. Maternal and perinatal outcome in cholestasis of pregnancy: a study in tertiary care hospital in North India. Int J Reprod Contracept Obstet Gynecol. 2018;7(12):5067.

15 Jaacks LM, Vandevijvere S, Pan A, McGowan CJ, Wallace C, Imamura F, et al. The obesity transition: stages of the global epidemic. Lancet Diabetes Endocrinol. 2019 Mar;7(3):231-40.

16 Estiu MC, Frailuna MA, Otero C, Dericco M, Williamson C, Marin JJ, et al. Relationship between early onset severe intrahepatic cho- lestasis of pregnancy and higher risk of meconium-stained fluid. PLoS One. 2017;12(4): e0176504.

17 Martineau M, Raker C, Powrie R, Williamson C. Intrahepatic cholestasis of pregnancy is associated with an increased risk of gestational diabetes. Eur J Obstet Gynecol Reprod Biol. 2014;176:80-5.

18 Marathe JA, Lim WH, Metz MP, Scheil W, Dekker GA, Hague WM. A retrospective cohort review of intrahepatic cholestasis of pregnancy in a South Australian population. Eur J Obstet Gynecol Reprod Biol. 2017;218: 33-8.

19 Brouwers L, Koster MP, Page-Christiaens GC, Kemperman H, Boon J, Evers IM, et al. Intrahepatic cholestasis of pregnancy: maternal and fetal outcomes associated with elevated bile acid levels. Am J Obstet Gynecol. 2015; 212(1): 100 .

20 Senocak GNC, Yilmaz EPT. Maternal and fetal outcomes in pregnancies complicated by intrahepatic cholestasis. Eurasian J Med. 2019 Oct:51(3):270.

21 American College of Gynecologists. ACOG committee opinion no. 560: medically indicated late-preterm and early-term deliveries. Obstet Gynecol. 2013;121(4):908-10.

22 Mullally BA, Hansen WF. Intrahepatic cholestasis of pregnancy: review of the literature. Obstet Gynecol Surv. 2002;57(1):47-52.

23 Saleh MM, Abdo KR. Intrahepatic cholestasis of pregnancy: review of the literature and evaluation of current evidence. J Womens Health. 2007;16(6):833-41.

24 Arrese M, Reyes H. Intrahepatic cholestasis of pregnancy: a past and present riddle. Ann Hepatol. 2006;5(3):202-5. 